\title{
A benchmark event sequence for mass ejection onset studies
}

\section{A flare associated CME with coronal dimming, ascending pre-flare loops and a transient cool loop}

\author{
R. A. Harrison and D. Bewsher \\ Space Science and Technology Department, CCLRC/Rutherford Appleton Laboratory, Chilton, Didcot, Oxfordshire, OX11 0QX, UK \\ e-mail: d.bewsher@rl.ac.uk
}

Received 25 July 2006 / Accepted 11 October 2006

ABSTRACT

\begin{abstract}
Aims. In this study, we report on the spectroscopic observations of a particularly well-observed flare and coronal mass ejection (CME) event sequence which we feel can be used as a benchmark study for CME onsets.

Methods. Specifically, we report on a set of extreme-ultraviolet (EUV) spectroscopic observations using the Solar and Heliospheric Observatory $(\mathrm{SOHO})$ to determine features of the $\mathrm{CME}$ onset process revealed through the analysis of plasma at different temperatures.

Results. The flare which occurred on the north-western limb was associated with a large CME. The event in question showed evidence for pre-flare ascending loops containing 1-2 million K plasma, which disappeared just prior to the flare. This disappearance is interpreted as coronal dimming, and it appears to coincide with the projected mass ejection onset time. In addition, a discrete, shortlived coronal loop containing plasma at transition region temperatures was detected just prior to this eruption. This loop displayed mass motion, along flux tubes, with oppositely directed flows. The nature and timing of this transient loop suggest a close relationship between it and the eruption process. Examinations of the timing and topology, which extend previous studies considerably, are found to be consistent with the mass ejection onset interpretation of Zhang and co-workers.

Conclusions. The clarity of this event sequence suggests that we should regard it as a benchmark in studies of the mass ejection onset process.
\end{abstract}

Key words. Sun: coronal mass ejections (CMEs) - Sun: flares - Sun: corona - Sun: UV radiation

\section{Introduction}

The coronal mass ejection (CME) onset process, and the nature of the flare-CME association have been closely studied for many years. There is no doubt that flares and CMEs are closely associated and the popular, though not universal, belief is that neither causes the other; they appear to be different manifestations of the same magnetic driver within different components of a magnetic system (see e.g. reviews by Harrison 2003, 2006, and references therein). However, the precise nature of the flare-CME relationship is not fully understood despite a number of years of multi-wavelength campaigns and analyses of CME onsets, using, for example, the Solar and Heliospheric Observatory (SOHO) spacecraft. There are a number of reasons for this. We observe CMEs using coronagraphs, which are best suited to the detection of events in the plane of the sky, i.e. the associated CME source regions will be near or on the limb, which means that studies which attempt to identify the CME source regions frequently suffer from foreshortening effects and even occultation by the solar disc itself. In addition, we must backproject CME events, through space and time, below the coronagraph occulting discs to identify the links to activity detected by instruments that image the solar disc directly. Thus, great care needs to be taken, and it has proven to be very difficult to obtain clear indicators of the CME onset process.

Given the importance of understanding CMEs to the investigation of coronal structure and evolution, as well as to space weather studies, an understanding of the CME onset process, including the links to flares, is a high priority for solar physicists.

One particular long-term effort, using the SOHO instrumentation has combined extreme-ultraviolet (EUV) and coronagraph observations in a series of approximately 200 campaigns run since 1996. The principal aim is to use spectroscopic information, using the SOHO Coronal Diagnostic Spectrometer (CDS, Harrison et al. 1995), of the source regions of CMEs in the low corona to obtain plasma parameters and structure and evolution information to shed light on the eruption process. A particular feature of these campaigns has been the detection and analysis of coronal dimming in specific emission lines. Results from these campaigns have been presented by Harrison and coauthors (Harrison 1997, 2003, 2006; Harrison \& Lyons 2000; Harrison et al. 2001, 2003; Howard \& Harrison 2004). These papers have presented studies of individual events or groups of events. One of the aims of the campaign was to build up observations of events in large enough numbers to perform statistical analyses, in particular to test issues such as the frequency of coronal dimming associated with CME onsets and the use of such phenomena for event prediction purposes (Bewsher \& Harrison 2006). Another aim is to identify events of special significance; some events may be particularly well observed.

Thus, we present here an analysis of the EUV signatures of a region on the solar north-west limb during the time of a CME eruption and flare. This is a particularly well observed sequence of events. The observations show pre-eruption, pre-flare 
ascending loops containing million $\mathrm{K}$ plasma as well as the brief appearance of a bright, large transition region temperature loop at the time of the CME onset. The loop appears to show evidence for counter-streaming plasma in a twisted structure, which overlies the subsequent flare site. The pre-event ascending loops disappear at the time of the CME onset and, indeed, their disappearance represents coronal dimming. The sequence of events is followed by a bright arcade EUV flare detected across a broad range of temperatures.

The pre-flare events of the sequence are found to support recent observations of ascending loops, and the disappearance of the loops provides support for the coronal dimming interpretations of recent CME-onset studies. The clear observation of a flare as part of this sequence allows a detailed study of the flareCME association as well as the CME onset process.

The event sequence in question occurred on 25 July 1999. Although the observations are not recent, we describe this event in detail now for a number of reasons. Some features of the event have been described by Harrison $(2003,2006)$ and Harrison et al. (2003). However, papers involving related observations of the same events have also appeared in the literature (Warren et al. 1999; Nitta et al. 2001; Kim et al. 2005) and these beg a more complete description of the events as detected using spectroscopic methods. Other more generic interpretations of CME onsets have also been published, e.g. Zhang et al. (2001, 2004); Gallagher et al. (2003); Qiu et al. (2004); Feynman \& Ruzmaikin (2004), which turn out to be well supported by the details of the 25 July events as seen with the CDS instrument, so it is timely to present these here.

In the next sections, we present the coronagraph and EUV observations, followed by an interpretation of the event sequence. Subsequently, we compare them to other CME onset and CME/flare studies. The relevance of the findings to specific CME onset models is discussed.

\section{Observations}

In the next 3 subsections, we describe the observations and findings of previous authors. Then we describe the details of the CDS spectroscopic observations.

\subsection{The $C M E$}

The CME of 25 July 1999 was detected using the LASCO (Large Angle Spectroscopic Coronagraph) coronagraph aboard SOHO (Brueckner et al. 1995). A sequence of fixed differenced images of the event are shown in Fig. 1. The white circle indicates the location and size of the solar disc. The LASCO data show a large loop-like CME originating from the solar north-west quadrant, with associated responses over a wide spread of position angles.

The event was first seen in images at 13:31 UT. It is clear that the principal event is a loop-like CME in the solar north-west, with a coincident, narrower eruption in the north-east. Responses to the eruptions can be seen in the running difference images as narrow black and white features as the streamers move.

The CME event is summarised by Harrison et al. (2003) (see their Table 4) as having a projected onset at 13:10 UT, a position angle spread from $232^{\circ}-360^{\circ}$ (angle from north, anticlockwise) and a mass of $3.5 \times 10^{12} \mathrm{~kg}$.

The time-height curve for the CME loop-front at that position angle is shown with stars $(*)$ in Fig. 2a (Data courtesy of Simon Plunkett, NRL). The event shows a rather fast constant velocity of ascent at $1118 \mathrm{~km} \mathrm{~s}^{-1}$, with a projected onset time
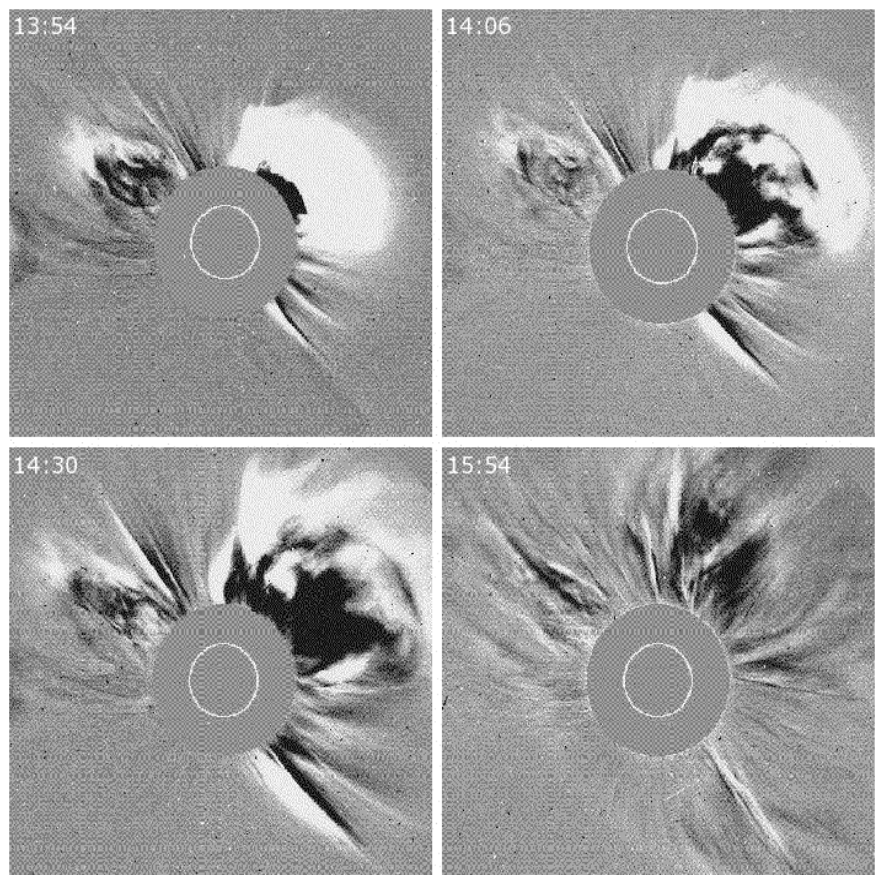

Fig. 1. LASCO C2 observations of the 25 July 1999 CME (Courtesy SOHO/LASCO team).

at 13:10 UT, assuming a zero-altitude limb onset and constant velocity.

Of course, there must be acceleration in the early stages of the eruption. This would place the CME onset at a time earlier than 13:10 UT. However, the CME source region must have an altitude greater than zero, which would suggest a later onset time, but if the source region is out of the plane of the sky, the onset would be earlier. Given all of these uncertainties, which are common to all CME onset analyses, we feel that we must use an onset window at least one hour wide. Thus, we use a CME onset window of 12:40 to 13:40 UT.

\subsection{The flare}

The flare is classified (Solar Geophysical Data 2000) as a GOES X-ray M2.4 event, as illustrated in Fig. 2c. It has a start time of 13:08 UT, peaking at 13:40 UT and slowly decaying to about 17:00 UT. In H-alpha, the event is recorded as occurring at 13:43 to $13: 46 \mathrm{UT}$, located in active region 8639 at N38 W81, and is a sub-flare (SF) event (Solar Geophysical Data 2000).

Although there is a $\mathrm{C}$ class flare visible in the GOES X-ray plot at 11:30 UT, there is no record of a $C$ class flare any where on the Sun at this time in the NOAA flare catalogue. To show that the flare did not occur in our region of interest, we plot the He I $(\diamond), \mathrm{OV}(+), \operatorname{Mg} \mathrm{IX}(\diamond)$ and Fe XVI $(\square)$ intensities across the whole of the CDS field-of-view (FOV) in Fig. 2d. There is no evidence of the $\mathrm{C}$ class flare in the CDS intensities.

There is also a "shoulder" in the GOES X-ray flux just before the onset of the flare at 13:08 UT. It is not known where this flux originated, but on comparison with the CDS intensities, it is unlikely to be from our field of view.

Warren et al. (1999) reported on TRACE (Transition Region and Coronal Explorer) and Yohkoh observations of the 25 July flare event. The principal features that they indicate are, the brightening of chromospheric ribbons early in the event, that are revealed to be the foot points of an arcade system detected later in the event at higher temperatures; the high-temperature loops 


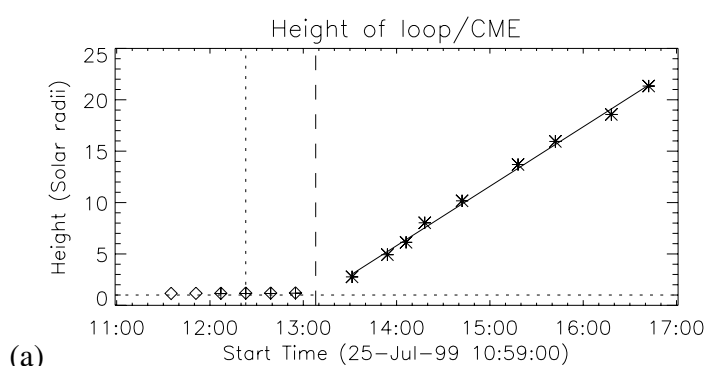

(a)

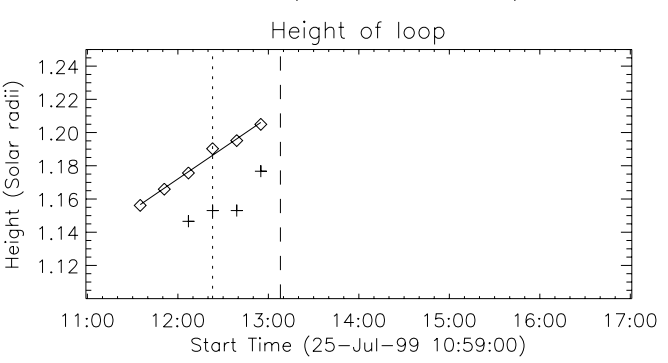

(b)

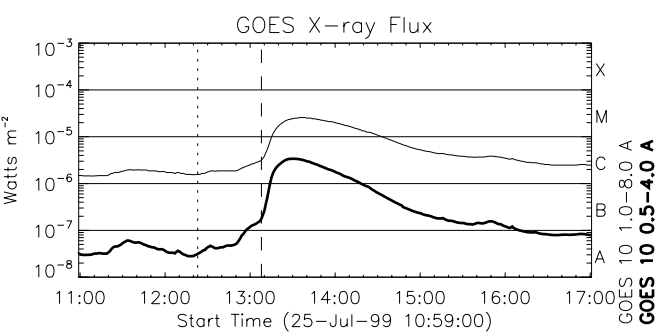

(c)

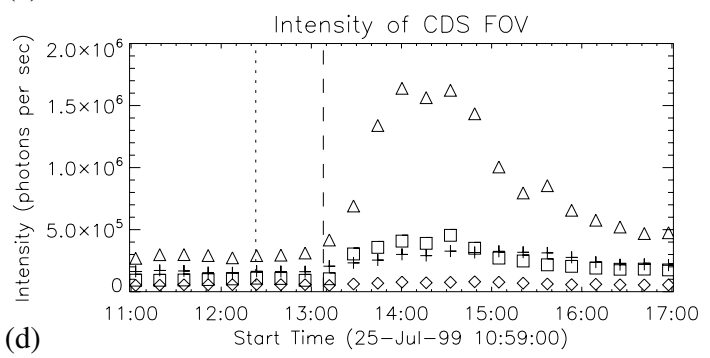

(d)

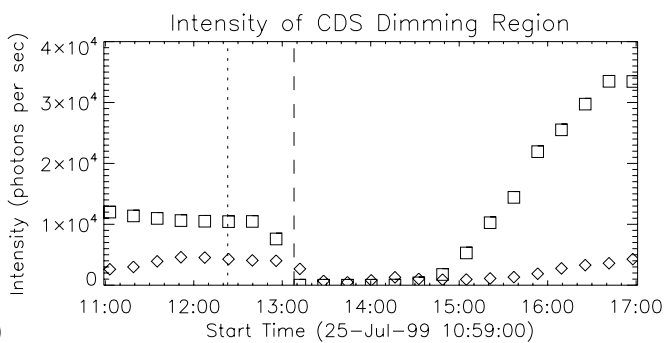

(e)

Fig. 2. a), b) Time-altitude profile of the Mg IX loop ( $)$, O v loop (+) and the principal loop front of the 25 July 1999 CME at position angle $300^{\circ}(*)$. c) GOES plot of the flare (Data courtesy NOAA). d) Total intensity of the CDS FOV for the He I $(\triangle), \mathrm{OV}(+), \operatorname{Mg} \mathrm{IX}(\diamond)$ and Fe XVI $(\square)$ emission lines. e) Intensity of the dimming region for the Mg IX $(\diamond)$ and Fe XVI ( $\square$ ) emission lines. In all frames, the vertical dashed line marks the time (13:08 UT) at which the X-ray plasma ejection occurred (Kim et al. 2005) and is the official start time of the GOES flare. The vertical dotted line marks the time (12:23 UT) when plasma flows were observed in the $\mathrm{OV}$ emission line.

form and some $1300 \mathrm{~s}$ later, cooler loops are seen to form below them.

The use of spectral data using CDS, described below, complements the data shown by Warren et al. (1999), by providing a range of intermediate temperatures not available to TRACE or Yohkoh, and by introducing capabilities for diagnostic analyses, investigating parameters such as flow speeds.

\subsection{Associated plasma ejections}

Kim et al. (2005) have analysed a large number of Yohkoh X-ray images to study the ejection of hot plasma in association with CMEs detected using SOHO/LASCO. They identified a range of event types. One event they studied, and illustrated in their paper, was that of 25 July 1999. Their Fig. 2 shows outward plasma motion in association with this event in the form of loops expanding at $112 \mathrm{~km} \mathrm{~s}^{-1}$ above the flare site, detected from 13:08 UT. They show a similarity between the topology of the plasma ejection and the CME structure, seen from 13:31 UT. This, they note, may suggest that the expanding X-ray loops may be the early signatures of the overlying CME.

\subsection{EUV spectroscopic observations}

The use of spectroscopic data from a wide range of emission lines (temperatures), from the $\mathrm{SOHO} / \mathrm{CDS}$ instrument, is now presented.

CDS is a spectrometer, which utilises the detection of spectral emission lines in the range 151-785 $\AA$ in order to determine coronal and transition region plasma diagnostic information. For the observation sequence used here, a $4^{\prime \prime} \times 240^{\prime \prime}$ slit was used, which was rastered over a $240^{\prime \prime} \times 240^{\prime \prime}$ field of view, using 60 exposures of $10 \mathrm{~s}$. Data from six emission lines were returned for each exposure, including spectral profiles. These emission lines (and the associated ion temperatures) were He I $584 \AA$ (20000 K), O v $629 \AA$ (250000 K), Mg IX $368 \AA$ (1 million K), Si X 347 and $356 \AA$ (1.3 million K) and Fe XVI $360 \AA$ (2 million $\mathrm{K})$. These data provide observations across a broad range of temperatures (20000 to 2 million K), including a wide range not covered in the studies mentioned above, and the Si X emission line pair can be used in ratio as a density sensitive indicator (Mason et al. 1997).

Only six emission lines were returned for each exposure because of the need to ensure a reasonable cadence in the face of limited telemetry. The resulting image cadence was $16 \mathrm{~min}$. CDS has $1.68^{\prime \prime}$ pixels along the slit, so the final images for this study are effectively $60 \times 143$ pixels of size $4^{\prime \prime} \times 1.68^{\prime \prime}$.

The CDS field of view was centred on 795" W, 602" N, of Sun centre. Thus the field is centred on a limb region at position angle $307^{\circ}$, with a view of the limb region from $300^{\circ}-310^{\circ}$. This is a very restricted field for comparing structures and events to CME onset activity; it is common to use larger area rasters for CME onset studies (e.g. Harrison \& Lyons 2000; Harrison et al. 2003). However, CDS was directed to active region 8639. The pointing and image size were consistent with those of the TRACE and Yohkoh data.

We do note immediately that given such small fields, comparisons to the large-scale structures of CMEs could be misleading. A CME may have a very large source region and the active region we are examining in the SOHO, TRACE and Yohkoh data may be much smaller than that source. In response, we point out that the events taking place in the active region are almost certainly associated with the CME and that despite the desire to cover a larger area, the results of this analysis will still prove to be useful in understanding the CME onset process. We also note that full-Sun imaging in instruments such as the SOHO EIT (Extreme Ultraviolet Imaging Telescope, Delaboudinière et al. 1995) show that this is the only site of major "surface" activities under the July $25 \mathrm{CME}$. Thus, the analysis, even within this restricted field, shows features, which appear to be very significant to the CME process. 

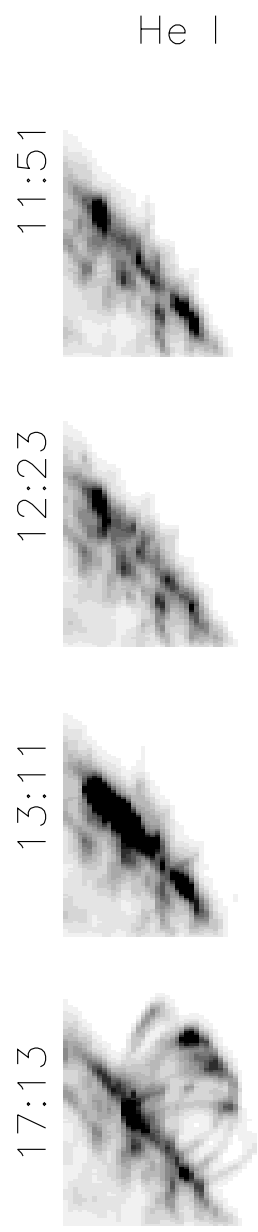
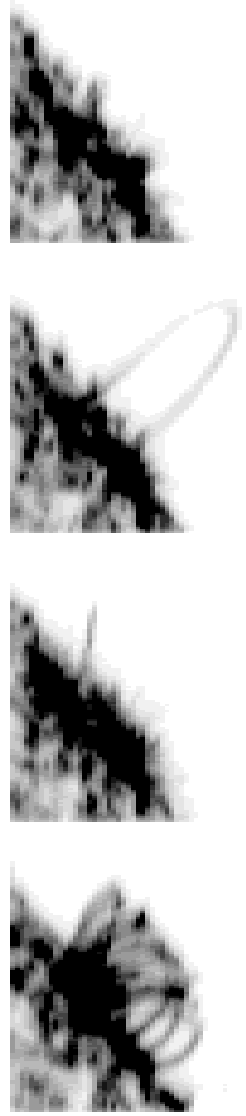

$$
\operatorname{Mg} \mid X
$$
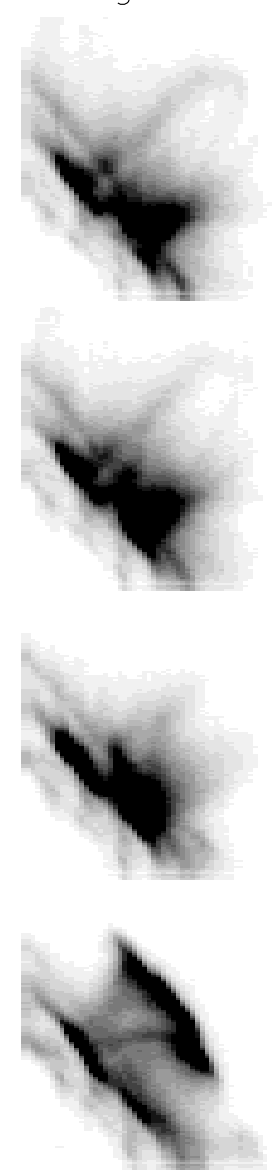

Si $x$
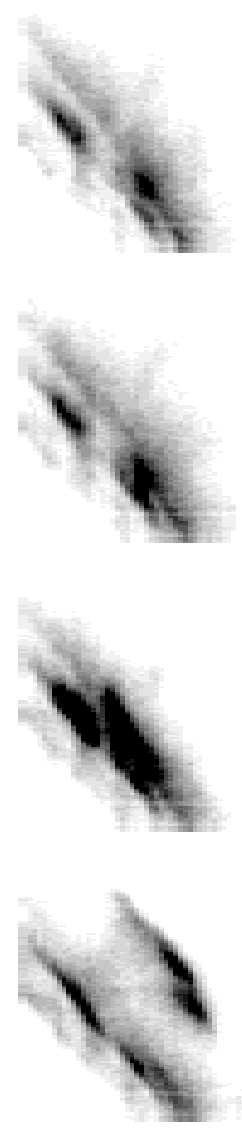

Si $x$
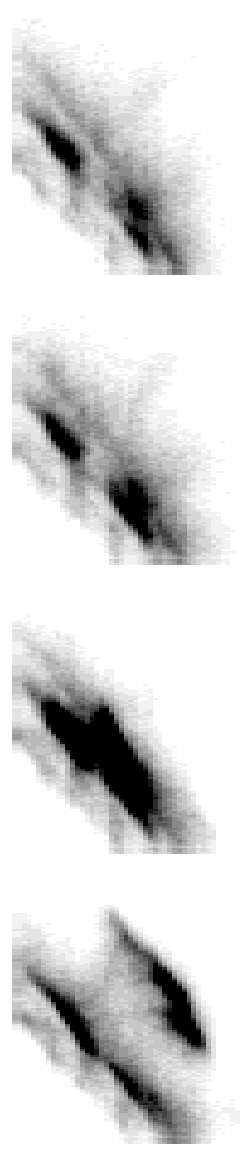

Fe XVI
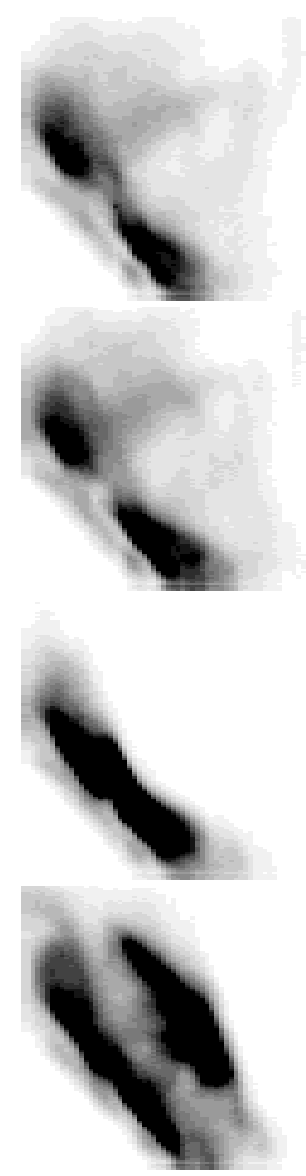

Fig. 3. Four snapshots of the July 251999 flare, taken at (top to bottom rows) 11:51, 12:23, 13:11 and 17:13 UT, illustrated in each case with images from the six emission lines used in the current study, from left to right He I $584 \AA$ ( $20000 \mathrm{~K}), \mathrm{O}$ V $629 \AA$ (250 $000 \mathrm{~K}), \mathrm{Mg} \mathrm{IX} 368 \AA$ ( 1 million K), Si X 347 and $356 \AA$ (1.3 million K) and Fe XVI $360 \AA$ ( 2 million K). The same $4^{\prime} \times 4^{\prime}$ field of view is imaged simultaneously in the six lines (temperatures). A reverse colour table has been used in this figure.

The CDS observations started at 11:03 UT and 24 rastered images were made, over a period of about $6 \mathrm{~h}$. For reference, we note that the CDS study number is 16947.

Figure 3 shows four of the rastered images, which we show at this stage for context information, to highlight the format of the data, the pointing, and the location of the flare. The top left image shows the He I data prior to the flare and running down the left hand column one can see the He I emission in the lead up to the flare, detected as a clear arcade in the final image. Note that this is a subset of the He I images taken. Similarly, the second column shows the OV emission which also reveals the arcade structure of the flare in the last frame. The hotter images show more diffuse off limb structure and, at these higher temperatures, the loops are less clear and we see a concentration of intensity due to line of sight effects along the top of the arcade. This view shows what many would call a classic post-flare loop system. To aid the later analysis one should note the location of the NW limb, clearly visible in the $\mathrm{He}$ and $\mathrm{O}$ images, which remains fixed for all of the images shown in this paper.

\subsubsection{Pre-flare ascending loop}

We start the analysis by examining the higher temperature plasma emission in particular prior to the flare onset. Figure 4 shows a composite of the first $16 \mathrm{Mg}$ IX million $\mathrm{K}$ images
(16 min cadence starting at 11:03 UT). The last 6-7 frames clearly show the flare as an over exposed bright region. These data have not saturated the CDS detectors, we have simply chosen a contrast to emphasise the weaker emission. Prior to the flare one can see weakly emitting structures in the corona with signs of changes between frames and some evidence for loops. Indeed, it has been claimed by Harrison $(2003,2006)$ that these pre-flare weakly emitting hot loops show signatures of expansion outward with time.

We clarify this point here by showing differenced images in Fig. 5, which are identical to Fig. 4 except that the intensity of the first frame is subtracted from all subsequent frames. This highlights changes in the solar atmosphere; white regions show enhanced emission with respect to the first frame and black show regions of depleted emission (dimming). Again, the flare is clearly visible as a saturated area but if one concentrates on the pre-flare images (the first 9), the evidence for weakly emitting pre-flare loops overlying the region is irrefutable.

Using the subtracted frames the outward expansion of the clearest white loop is measured and the loop top is plotted in Fig. $2 \mathrm{~b}$ with diamonds $(\diamond)$. The data from the 3rd-8th frames clearly show the loop and these data are used in Fig. 2b. The absolute error on these points would be due to the clarity of the loop itself, which is approximately $6500-7000 \mathrm{~km}$ thick at the loop top, and to the error on the location of the limb, but 

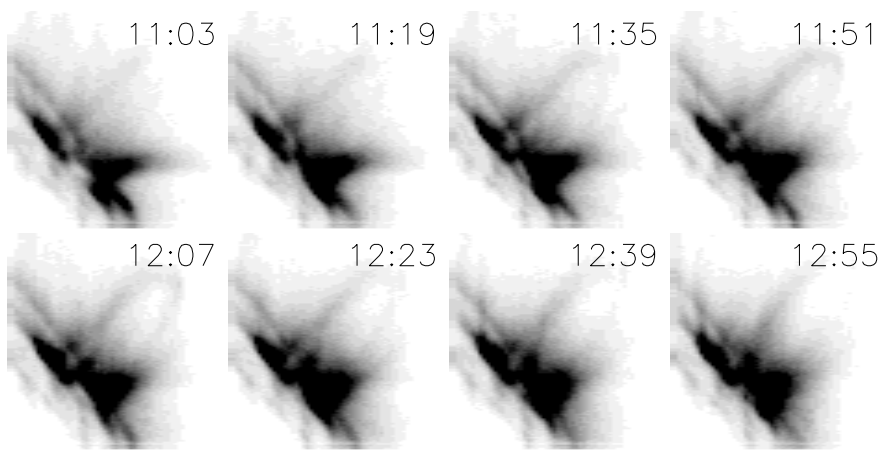

$13: 11$
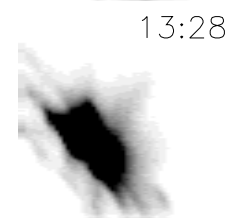

$14: 16$

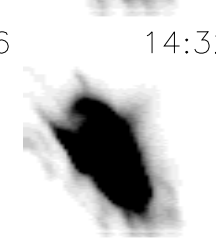

$14: 00$
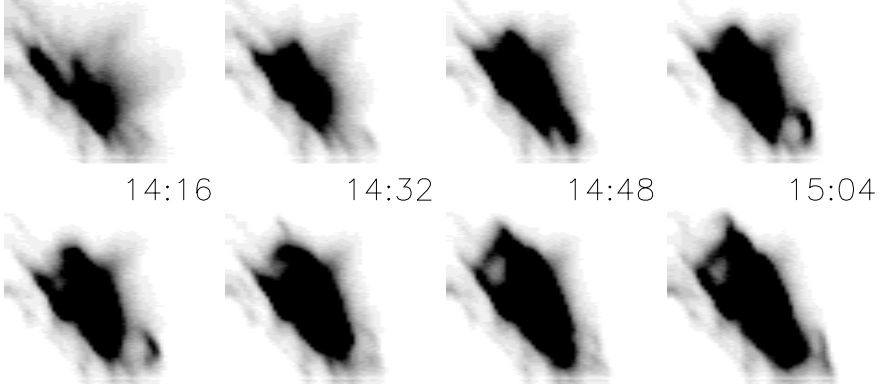

Fig. 4. The first 16 images shown in the Mg IX $368 \AA$ emission line. Each frame shows an area $4^{\prime} \times 4^{\prime}$ with the top left frame taken at 11:03 UT and time running from top left to bottom right, with $16 \mathrm{~min}$ cadence.

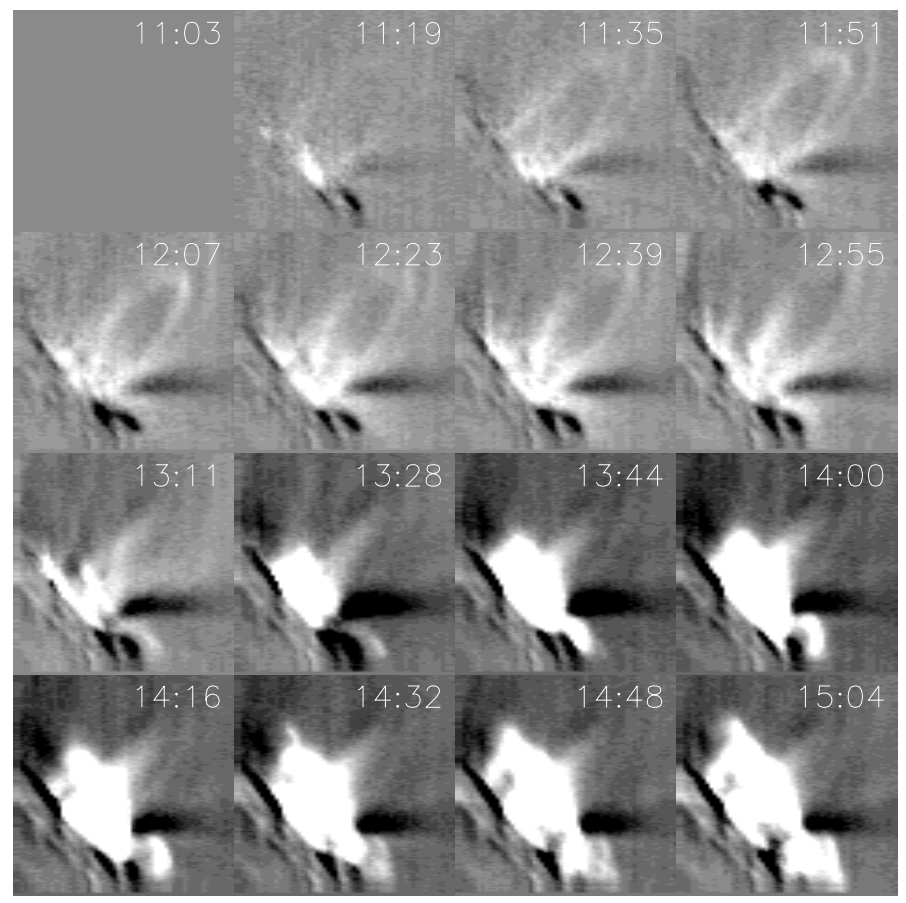

Fig. 5. The first $16 \mathrm{Mg}$ IX frames with the first frame subtracted to highlight changes in the corona.

there is a clear ascent. Also shown in the plot is a solid line fitted to the data, which has a gradient of $7 \mathrm{~km} \mathrm{~s}^{-1}$. It should be noted that we see evidence for further loops, such as an outer loop overlying the loop for which we have plotted the height profile.

At $7 \mathrm{~km} \mathrm{~s}^{-1}$ any consideration of back-projection becomes rather academic. If we were to insist on a projected onset from the active region at that speed, to zero altitude, we would be talking of an onset at about 08:00 UT. If we consider any starting
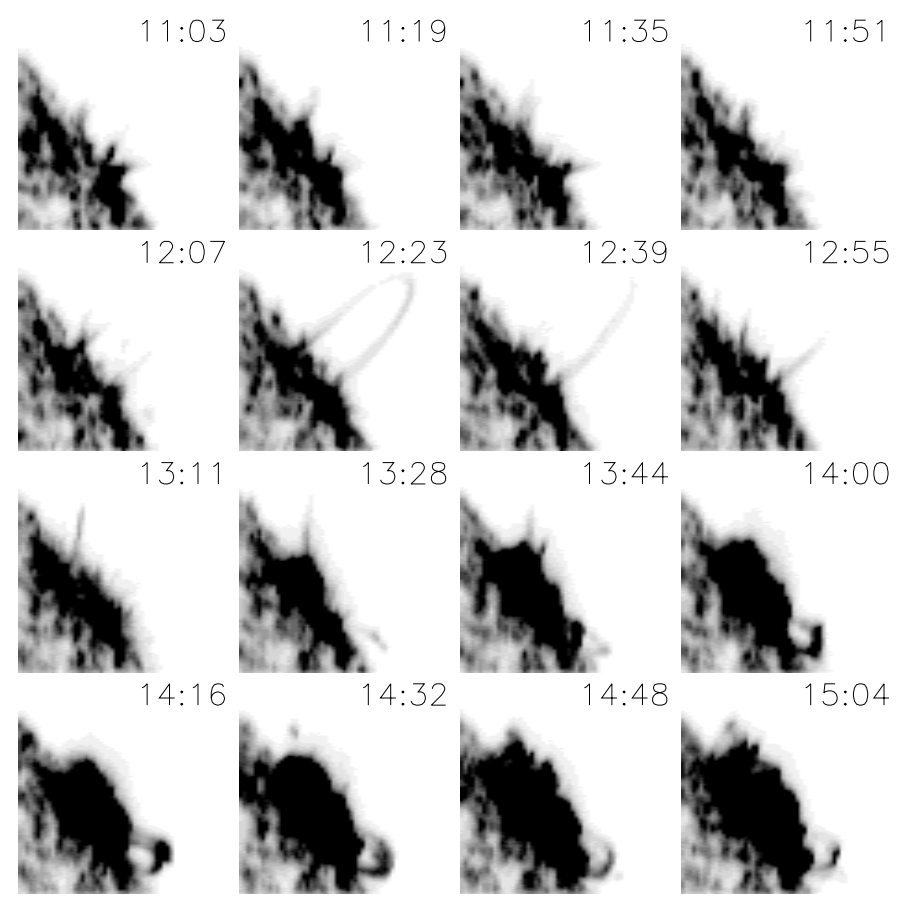

Fig. 6. The first sixteen images, as with Fig. 4, but this time taken in the $250000 \mathrm{~K} \mathrm{O} \mathrm{V} \mathrm{emission} \mathrm{line} \mathrm{at} 629 \AA$.
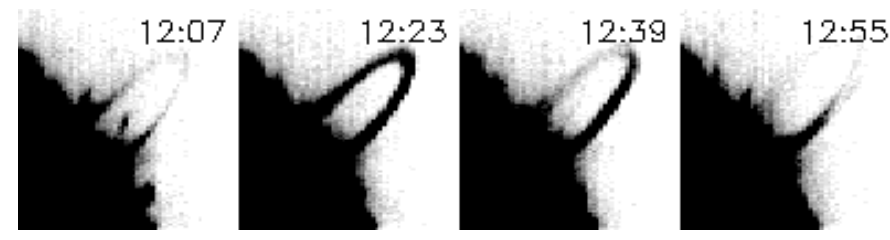

Fig. 7. Frames 5-8 of Fig. 6 with the contrast altered so that the top of the $\mathrm{O} v$ loop can be clearly seen.

altitude, the onset would be considerably later. However, at these speeds one has to start asking questions like - what is the difference between eruption and magnetic evolution, with such slow speeds of ascent? Given this, the most productive conclusion here is to state simply that we see slowly ascending loops in the early, pre-flare phase of this event.

\subsubsection{Pre-flare flow pattern in cool loops}

There is one other feature of interest which we highlight here, which was first reported by Harrison (2006), but little analysis has been reported to date. The feature is a loop, evident in the transition region temperature of $250000 \mathrm{~K}$, viewed in the $\mathrm{O} \mathrm{V}$ $629 \AA$ emission line for just a few frames. This loop is the principal feature of frames 6 and 7 of Fig. 6, and the feet of the loop are also visible in frames 5 and 8 . The loop achieves altitudes of about $100000 \mathrm{~km}$ and it appears for a period of 45-60 min prior to the flare.

Figure 7 shows frames 5-8 of Fig. 6 with the contrast altered so that the top of the $\mathrm{O} v$ loop can be clearly seen. The altitude of the loop top is also displayed in the time-height curve of Fig. 2b, shown with a cross (+). This loop is clearly not identical to the earlier ascending loop and shows marginal evidence of ascent.

This cool loop may only exist for a short time but it has remarkable features. One of these is its velocity structure, shown in Fig. 8. A raw frame is shown on the left hand side of this figure and the corresponding Doppler map is shown. Whilst 


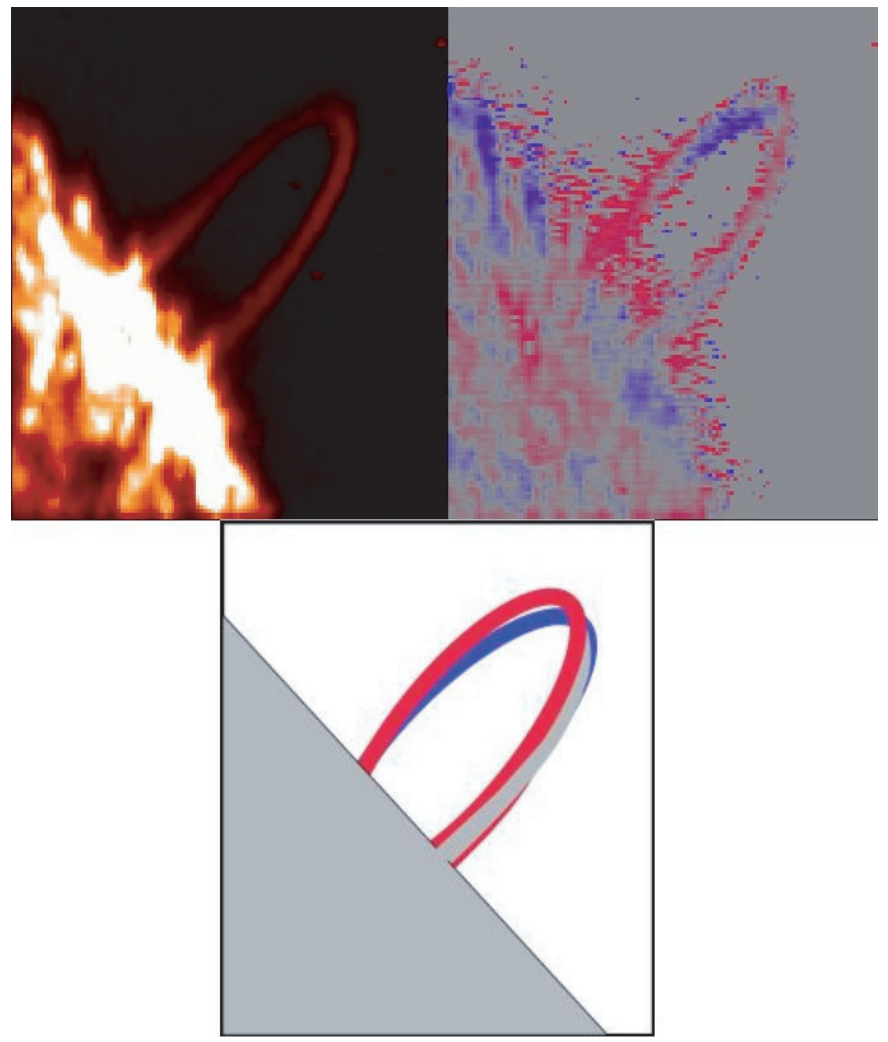

Fig. 8. The "cool" O v loop shown in intensity and as a Doppler map, and a schematic image of the probable topology (see text).

spectroscopic data has a powerful ability to provide velocity information, there is always the issue of the identification of the "rest" position. Calibration of the CDS instrument cannot be done effectively using cool lines as done using some longer wavelength instruments, so we are reliant on calculation and some common sense argument. For the image of Fig. 8 the line centre (at rest) has been set to a calculated location and the image inspected to ensure the lowest level of net motion on the disc; at any point in time as much material is presumably moving away from you as towards you. However, even with generous variations in the rest position, the basic flow structure of the loop itself remains the same and it is only the absolute velocity levels that one should question.

The cool, O V loop shows both red and blue shifted components. The left hand side of the loop, near the foot point, shows a significant red shifted region. Just to the left of the loop-top, we see a bright blue-shifted region. To see both red and blue shifted material within what appears to be one loop is almost impossible to visualise when one considers the geometry. Thus, the most logical conclusion is that the loop is made up of flux-tubes with differing flow patterns. The cartoon at the bottom of Fig. 8 is intended to explain in the simplest form the possible layout. If there are flows in a single loop with a line of sight component, the loop will almost always appear as just red or blue. Consider a red-shifted flux tube as drawn. Suppose that there is a second flux tube with material flowing the other way - i.e. blue shifted - overlapping, as sketched so that the red shifted loop foot point is closer to the observer on the left hand side. The two flux-tubes are slightly tilted to reveal the blue-shifted region to the left of the loop top, as in the data. Finally, the data show a "grey" lane in the right hand leg of the loop, with red shifted material on either side. This can be explained by suggesting that the "blue" loop has a geometry such that the right hand foot point has no

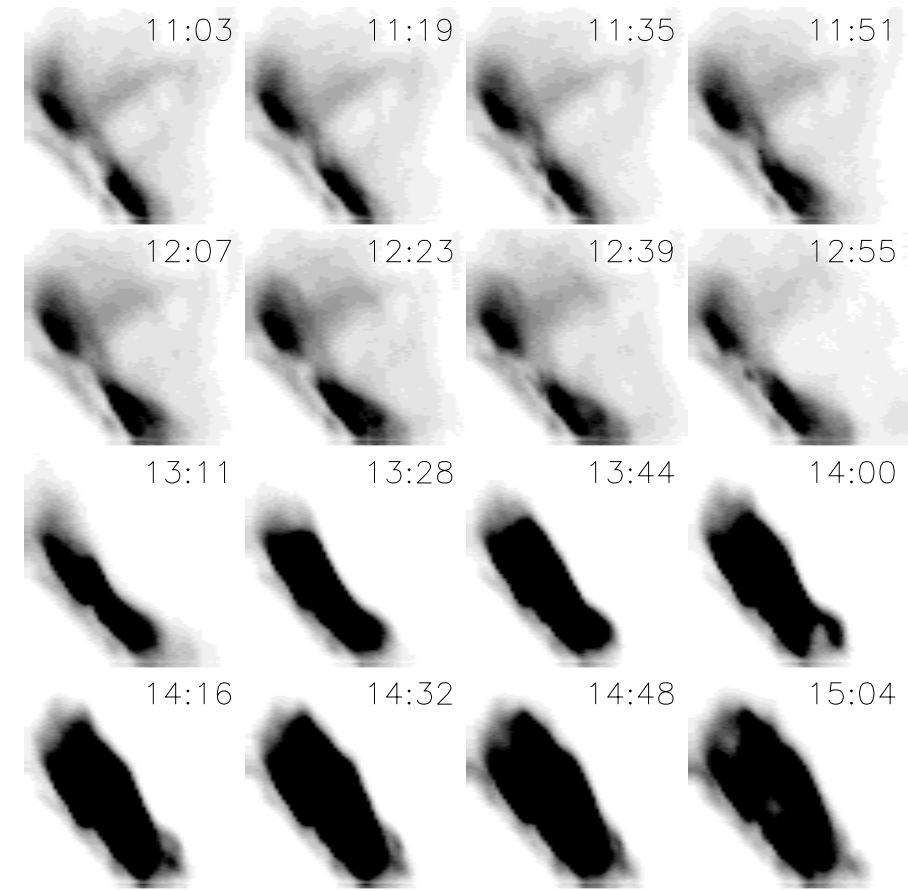

Fig. 9. A composite of the images in the same format as Figs. 4 and 6, in raw intensity images, for the 2 million K Fe XVI emission line at $360 \AA$.

line of sight component to the observer. Thus, in cartoon form, this leg is shown grey in the right hand image.

It is interesting to note that this interpretation is revealing three-dimensional aspects of the geometry and such interpretation could be used to model this event further. However, the relevant features for this report are:

- we see a short-lived "cool" coronal loop, which apparently ascends behind the previously identified hot loops;

- there is evidence for flows, which suggest streaming in differing directions along different flux-tubes.

\subsubsection{Coronal dimming}

We now turn to the issue of coronal dimming, in association with CME onsets. This is an area which has been reviewed recently (Harrison 2006) and indeed the event in question has been analysed in this respect by Harrison et al. (2003). The striking feature of this event is shown in Figs. 2e and 9. Figure 9 shows the same 16 images as for the other emission lines, shown above, but for the Fe XVI $360 \AA$ emission line, characteristic of 2 million K. The contrast has been chosen to specifically identify diffuse, weak features, so the flare, which is evident in the last 7-8 images is, again, saturated. In the earlier frames we can see the ascending hot loops which were described in the million $\mathrm{K} \mathrm{Mg} \mathrm{IX} \mathrm{emis-}$ sion, Fig. 4, above. These loops are diffuse but nevertheless quite evident. However, between the 8th and 9th image, the off limb corona shows a substantial drop in intensity. The loops disappear. Figure 2e shows the intensity of the corona drop by $89 \%$ and $42 \%$ in the Fe XVI and Mg IX emission lines, respectively, as shown in Fig. 6 of Harrison et al. (2003).

A Si X line ratio density diagnostic also available in the data set was used by Harrison et al. (2003) to calculate a $94 \%$ density decrease (from $\log _{10}=9.4 \mathrm{~cm}^{-3}$ to $\log _{10}=8.2 \mathrm{~cm}^{-3}$ ) at a temperature of 1.3 million $\mathrm{K}$. This density decrease was subsequently shown to be evidence for the probable loss of up to $3.4 \times 10^{12} \mathrm{~kg}$ of matter from the imaged region and good 
evidence for the suggestion that the dimming is due to the loss from the corona of loops containing hot plasma.

\section{Further interpretation}

The first point to note is the relative timing of the features revealed here. Let us consider the relative timing of the events discussed:

- $11: 19$ to $12: 55 \mathrm{UT}$ - we detect weak, diffuse 1-2 million K (Mg IX and Fe XVI) coronal loops ascending above the active region at $7 \mathrm{~km} \mathrm{~s}^{-1}$, detected from altitudes of $100000 \mathrm{~km}$.

- From 12:07 to 12:55 UT - we identify a discrete transient transition region temperature $(250000 \mathrm{~K})$ loop $(\mathrm{O} \mathrm{v})$ at coronal heights (100000 km altitude) and showing evidence for ascent. This loop shows evidence for plasma flows of differing directions in different flux-tubes.

- X-ray plasma ejection observed at 13:08 UT (Kim et al. 2005).

- Official start of the GOES X-ray flare at 13:08 UT.

- Coronal dimming begins between 12:55 and 13:11 UT in both the Mg IX and Fe XVI emission lines. During this period, the loops observed disappear. It is the disappearance of these loops that constitutes the coronal dimming phenomenon.

- The images (frame 10 of Figs. 4 and 9) reveal a flare event certainly from the observation at 13:28 UT though the starttime is somewhat subjective and the CDS data have a cadence of $16 \mathrm{~min}$. The GOES start time is listed at 13:08 UT.

- We note that the projected CME onset, using LASCO data was 13:10 UT, though the back projection must introduce an uncertainty as discussed above of maybe $\pm 30 \mathrm{~min}$ or more.

Before interpreting this further, we make note of two important analyses whose conclusions are relevant to this work, namely the papers by Zhang et al. (2001, 2004), which attempt to bring together a unique set of multi-wavelength observations for a set of CMEs. They use data from instruments on SOHO, as well as the GOES X-ray data of observations made of CMEs originating near the solar limb. However, the key feature of their studies is the use of the LASCO C1 coronagraph. $\mathrm{C} 1$ is the innermost portion of the LASCO coronagraph, which was lost in mid-1998. It allowed observations down to $1.1 R_{\odot}$ from Sun-centre. Thus, by far the majority of LASCO data-sets used for onset studies are restricted to an inner limit of $2.0 R_{\odot}$ (the $\mathrm{C} 2$ inner edge) because the loss of $\mathrm{C} 1$ occurred prior to the build up of $\mathrm{CME}$ activity for the last solar maximum. There are few events where we have clear $\mathrm{CME}$ observations down to $1.1 R_{\odot}$ combined with "surface" observations in X-rays or/and the EUV, so the studies of Zhang et al. $(2001,2004)$ are particularly important both to investigate the lowest altitude activity and to test our understanding of CME onsets and the CME-flare question.

The time-altitude curves for three of the four events reported by Zhang et al. (2001) show a three-phase ascent, specifically, (i) an "initiation phase", which displays a gradual expansion in the CME loops at speeds of under $80 \mathrm{~km} \mathrm{~s}^{-1}$ (the events they studied showed the initiation phases lasting from half an hour to 2 hours), (ii) an "impulsive acceleration" phase during which there is a sudden increase in the CME speed of ascent, at altitudes in the range 1.3-4.6 $R_{\odot}$ above Sun-centre (for their events this coincided in time with the onset and rapid rise in intensity of an associated flare); and (iii) the "propagation phase", where the events ascend at constant or near-constant velocities.
The conclusions of Zhang et al. $(2001,2004)$ are the following:

- CMEs and flares are two different manifestations of the same magnetic process;

- They have a strongly coupled relationship but not a cause and effect one.

These, they point out, are consistent with the interpretation of Harrison (1995). The results reject the scenario where CMEs are driven by flare-induced coronal responses, because the initiation phase of the CME is clearly pre-flare. They suggest that the initiation phase may be caused by the destabilisation and quasi-static evolution of a large-scale coronal magnetic structure. They argue that if a critical point is reached, violent magnetic activity may be triggered that induces the magnetic force to drive the $\mathrm{CME}$, whilst simultaneously triggering the flare.

The ascending 1-2 million K loops of the 25 July observations appear to be consistent with the Zhang et al. (2001, 2004) initiation phase, i.e. evidence for ascending structures which are clearly pre-flare and may precede the apparent back projected CME onset time. We do not have $\mathrm{C} 1$ observations of the July events but the altitudes in question are consistent. The disappearance of the 1-2 million $\mathrm{K}$ loops, as the coronal dimming, between 12:55 and 13:11 UT is consistent with a rapid ascent phase, as is the observation of the ascent of the $\mathrm{OV}$ loop at 12:55 UT. The flare becomes evident in the images in the following frames. Thus, we find agreement between the Zhang et al. $(2001,2004)$ impulsive acceleration phase and the onset of dimming, yet we still argue for a pre-flare onset to the sequence of events - as does Zhang et al. (2001, 2004). The reason for the discrete $\mathrm{OV}$ coronal loop is less clear and is not apparently related to any aspect of the Zhang et al. $(2001,2004)$ work, though such a cool feature would not have been detected in the $\mathrm{C} 1$ data. This cool loop is a temporary feature showing a phase of mass flow in flux tubes at about the time leading the impulsive acceleration phase. Thus, it may be that the OV loop denotes a key feature of the sequence of events, in that it signals the onset of the instability or lack of equilibrium. It may represent the lowest levels of the erupting loops in whose flux tubes we see mass motion as a result of siphon flows due to the pressure differences driven by the changing magnetic configuration in the lead up to the eruption. The sudden, transient nature of this activity strongly suggests that this may be the case. If this is so, for this event we may not only be witnessing a signal of the impending impulsive acceleration phase or onset of instability or lack of equilibrium, but we may be identifying the delimiter of the region of erupting fields, i.e. the boundary between those fields which are erupting and those that remain closed.

Returning to the work of Kim et al. (2005) on the July 25 event, their outward expanding X-ray loops were travelling at $112 \mathrm{~km} \mathrm{~s}^{-1}$ and were detected from 13:10 UT. The ascending EUV loops we report here, using the Mg IX and Fe XVI observations were clearly detected pre-flare at much slower speeds and had erupted out of the field of view by 13:08 UT. It is conceivable that these are the same loop systems, though there is no observational overlap. However, as with Kim et al. (2005), we note the consistency with the location and topology of the plasma and the overlying CME.

Although this work is mostly concerned with the pre-flare initiation activities, we note that the images of Fig. 3 show great consistency with the flare observations of Warren et al. (1999). Compare their Fig. 2 with our Fig. 3, above. The CDS He I and TRACE H I (1216 А) images show similarities, as do the TRACE iron data at $171 \AA$ and $195 \AA$, when compared to the $\mathrm{Mg}$ 
IX CDS images. Particularly useful additions to these flare studies are the pre-flare 1-2 million $\mathrm{K}$ ascending loops and the transition region temperature data, in particular the transient $\mathrm{O} V$ loop.

It is also worth noting here, that the studies by Feynman \& Ruzmaikin (2004); Gallagher et al. (2003); Qiu et al. (2004) all show evidence of the three phase CME propagation suggested by Zhang et al. (2001) using data from a variety of space based instrumentation. The study by Qiu et al. (2004) also supports the idea of CME onset before the flaring activity. Finally, it is also noted that studies of preflare CME onset have been reported for many years with Harrison (1986) being an early example.

\section{Final comments}

It is clear that the well-observed sequence of events of 25 July 1999 show a number of useful aspects which are highly relevant to the CME and flare onset question, despite the rather restricted fields of view of the SOHO and, indeed, the TRACE, observations of this event. Revisiting this event sequence now, with the first complete report of the CDS/SOHO data has allowed a better understanding of a number of previous reports and is highly relevant to the work of Zhang et al. $(2001,2004)$. Thus, this has been a timely study. It has highlighted:

- the importance of pre-flare activity, namely pre-flare, ascending coronal loops, in particular in relation to CME onsets;

- an example of one of the clearest interpretations of coronal dimming;

- an intriguing feature, namely the detection of a discrete, short lived coronal loop in transition region temperatures, with oppositely directed mass flows, at the onset of the impulsive acceleration phase.

The potential importance of this last feature has been stressed as an indicator of the onset of instability or equilibrium loss, and possibly of the delimiter between erupting and closed fields, and, thus, other features of this kind will be sought in the numerous CME onset studies made using the SOHO CDS instrument.

Acknowledgements. SOHO is a mission of international cooperation between ESA and NASA. CDS was built and is operated by a consortium led by the Rutherford Appleton Laboratory and including the Mullard Space Science Laboratory, the NASA Goddard Space Flight Center, Oslo University and the Max-Planck-Institute for Extraterrestrial Physics, Garching.

D.B. would like to thank the UK's Particle Physics and Astronomy Research Council for financial support. The authors would also like to thank the referee whose comments helped to increase the clarity of the paper.

\section{References}

Bewsher, D., \& Harrison, R. A. 2006, A\&A, in preparation Brueckner, G. E., Howard, R. A., Koomen, M. J. et al. 1995, Sol. Phys., 162, 357 Delaboudinière, J.-P., Artzner, G. E., Brunard, J. et al. 1995, Sol. Phys., 162, 291 Feynman, J., \& Ruzmaikin, A. 2004, Sol. Phys., 219, 301

Gallagher, P. T., Lawrence, G. R., \& Dennis, B. R. 2003, ApJ, 588, L53

Harrison, R. A. 1986, A\&A, 162, 283

Harrison, R. A. 1995, A\&A, 304, 585

Harrison, R. A. 1997, Proc. 31st ESLAB Symp. Correlated Phenomena at the Sun, in the Heliosphere and in Geospace, ESA SP-415, 121

Harrison, R. A. 2003, Adv. Space Res. 32(12), 2425

Harrison, R. A. 2006, AGU Monograph Solar Coronal Mass Ejections and Energetic Particles, in press

Harrison, R. A., \& Lyons, M. 2000, A\&A, 358, 1097

Harrison, R. A., Sawyer, E. C., Carter, M. K. et al. 1995, Sol. Phys., 162, 233

Harrison, R. A., Bryans, P., \& Bingham, R. 2001, A\&A, 379, 324

Harrison, R. A., Bryans, P., Simnett, G. M., \& Lyons, M. 2003, A\&A, 400, 1071

Howard, T. A., \& Harrison, R. A. 2004, Sol. Phys., 219, 315

Kim, Y.-H., Moon, Y.-J., Cho, K.-S., Kim, K.-S., \& Park, Y. D. 2005, ApJ, 622,1240

Mason, H. E., Young, P. R., Pike, C. D. et al. 1997, Sol. Phys., 170, 143

Nitta, N. V., Sato, J., \& Hudson, H. S. 2001, ApJ, 552, 821

Qiu, J., Wang, H., Cheng, C. Z. et al. 2004, ApJ, 604, 900

Solar Geophysical Data, 2000, No. 665, Pt. II

Warren, H. P., Bookbinder, J. A., Forbes, T. G. et al. 1999, ApJ, 527, L121

Zhang, J., Dere, K. P., Howard, R. A., Kundu, M. R., \& White, S. M. 2001, ApJ, 559,452

Zhang, J., Dere, K. P., Howard, R. A., \& Vourlidas, A. 2004, ApJ, 604, 420 